\title{
Diagnóstico de la gestión de derrames de hidrocarburos en gasolineras
}

\author{
María Victoria Vaca Molina / Evelyng Astudillo Sánchez
}

\section{Resumen}

El comercio de combustibles, actividad económica esencial en Ecuador, tiene como riesgo principal el derrame de combustibles. El objetivo del estudio fue diagnosticar la situación de las gasolineras en la ciudad de Guayaquil. Mediante la inspección de 43 gasolineras en esta ciudad y a través de entrevistas a expertos, se determinaron características comunes de las gasolineras, medidas de seguridad, causas de derrames, criterios prioritarios para elegir técnicas de remediación y de estas las más utilizadas. Se encontró que las gasolineras almacenan un promedio de 36.514 galones de combustible y cuentan con las herramientas necesarias para prevenir y detectar derrames. Se identificaron los criterios eficacia y tiempo como prioritarios para elegir una tecnología de remediación. Los métodos más utilizados son los físico químicos.

\section{Palabras clave:}

Hidrocarburos, técnica de remediación, restauración, derrames, gasolinera

\section{Abstract}

The marketing of fuels is an essential economic activity in Ecuador, fuel spill-overs are its main risk. The aim of the study was to assess the situation of gas stations in Guayaquil. By inspecting 43 gas stations and interviewing experts, common characteristics of gas stations, security measures, and causes of spills, priority criteria for choosing remediation techniques and the most used techniques were determined. It was found that gas stations store an average of 36514 gallons of fuel and have the tools necessary to prevent and detect spills. Efficiency and time were identified as priority criteria for choosing remediation technology. The most widely used technology is physical chemical methods.

\section{Keywords:}

Hydrocarbon, remediation techniques, restauration, spill- overs, gas stations. 


\section{Introducción}

Una de las características del mundo actual y globalizado es que las personas necesitan movilizarse continuamente para cumplir con sus actividades diarias, es por eso que continuamente se están desarrollando métodos de transporte cada vez más rápidos y seguros. Dentro de esta carrera las gasolineras cumplen con la función de abastecer de combustible a los diferentes tipos de transporte terrestre (Cerón, 2010). Sin embargo, a pesar del importante rol de las mismas estas han operado sin cumplir ciertas normas técnicas de funcionamiento, especialmente normas ambientales y de seguridad. Por lo que, debido a esto, uno de los riesgos inminentes dentro de las gasolineras en nuestro país es el derrame de hidrocarburos (Cortázar, 2013).

En Ecuador, entre las posibles causas de derrames de combustibles en las gasolineras está la falta de actualización en tecnologías de seguridad, ya que muchas de las gasolineras existen desde antes de 1995, por lo que no poseen los nuevos sistemas de seguridad (La Hora, 2012). Otra causa es la planeación deficiente de las empresas, sobre todo por la falta de responsabilidad social ambiental en los directivos, la carencia de previsión futura y la insuficiencia de personal especializado que puedan reducir los riesgos de derrames contaminando subterráneamente el agua y suelo que se aprovechan en las ciudades.

No obstante, países como Chile, Estados Unidos, México, Colombia y otros similares en Europa no son ajenos a los inconvenientes generados por la contaminación de suelos con hidrocarburos (López, Santana, Rodríguez, \& Mesa, s.f.).

Considerando el desarrollo de las actividades hidrocarburíferas en nuestro país, es importante tomar en cuenta el riesgo inminente que significan actividades aparentemente sencillas, como las de almacenamiento y comercialización de combustibles en ciudades. Este trabajo pretende realizar un diagnóstico de la situación actual de las gasolineras en la ciudad de Guayaquil, incluyendo las medidas de seguridad para prevención y detección de derrames y las posibles estrategias de remediación a aplicarse. Además, servirá como punto de partida para investigaciones más a fondo de las medidas de seguridad para el control de la contaminación ambiental, asociado al funcionamiento de las gasolineras.

\section{Marco teórico}

\section{Extracción y comercialización de} combustibles

La Empresa Pública de Hidrocarburos del Ecuador, EP PETROECUADOR, creada mediante Decreto Ejecutivo 315 del 14 de abril del 2010, es la empresa encargada de la producción de derivados de petróleo para la comercialización interna y exportación. En el año 2013 generó ventas de 16,334.49 millones de dólares, colocándola en la posición número uno de las mayores empresas del país (Revista Vistazo, 2013).

Los derivados de petróleo mayormente comercializados por gasolineras son súper, extra, ecopaís; además de diésel, fuel oíl, jet fuel, gas licuado del petróleo (GLP) (Dirección de análisis estadístico $\mathrm{y} / \mathrm{o}$ indicador, 2015).

El proceso de comercialización de estos derivados comprende las siguientes fases: transporte, almacenamiento, abastecimiento, distribución y venta al usuario. La EP PETROECUADOR, se encarga desde la primera fase hasta la distribución a diferentes comercializadoras que venden el producto a los clientes finales (Agencia de Regulación y Control Hidrocarburífero, 2015). La comercialización o venta de estos productos se refiere a todas las actividades destinadas al suministro de los mismos, al consumidor final y en estas actividades se incluyen, tanto a distribuidores mayoristas como minoristas, encontrándose las ga- 
solineras en este último grupo.

Antes del proceso de comercialización y luego del proceso de refinación, los derivados de petróleo son transportados hasta las terminales de combustibles de EP PETROECUADOR, para su posterior almacenamiento y venta al por mayor. El transporte se realiza por medio de unidades automotrices destinadas al transporte terrestre de combustibles, llamadas auto tanques, tracto camiones o tanqueros. En el caso de Guayaquil, la terminal más cercana es la Terminal Pascuales. Una vez que los combustibles se encuentran almacenados en los tanques estacionarios de las terminales, es distribuido por medio de auto tanques a las instalaciones de cada cliente para descargarlo en sus tanques. Estos clientes son regularmente gasolineras, que a su vez, venderán los combustibles al consumidor final (venta al por menor) (Ministerio del Ambiente, 2011). En la Figura 1, a continuación se presenta un esquema del proceso.

\section{Figura 1.}

Proceso de comercialización de combustibles

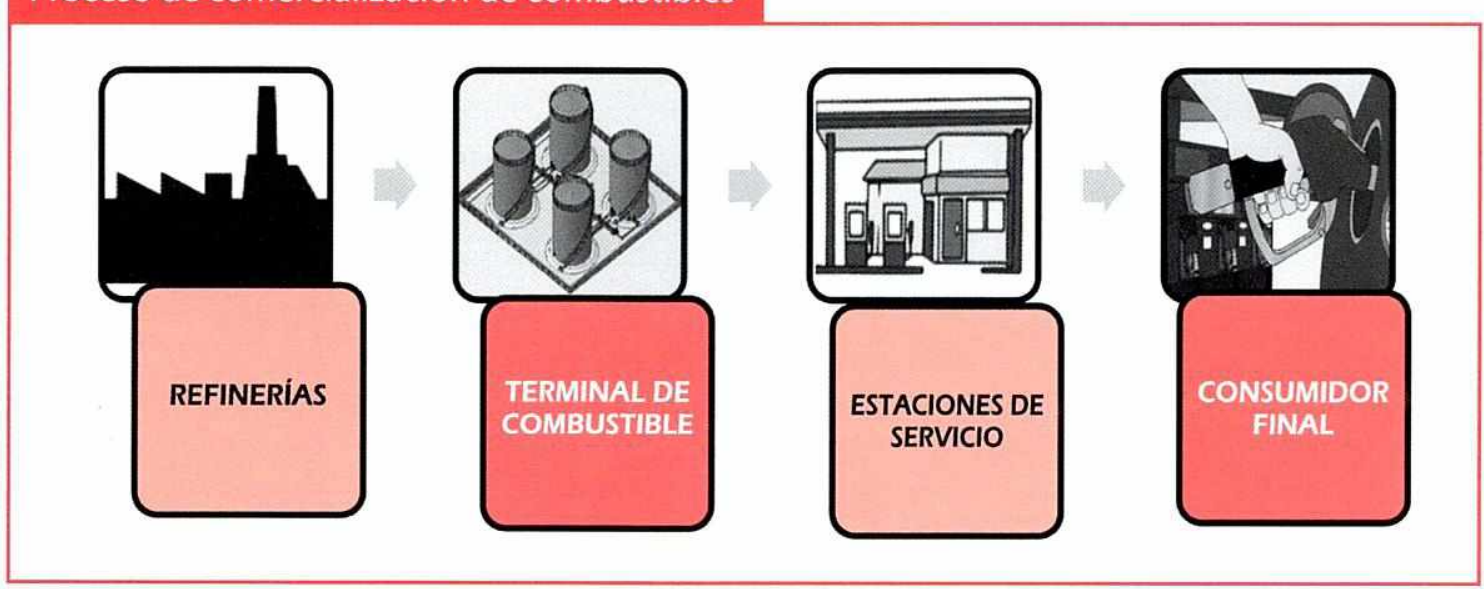

Fuente: La Autora

En el cantón Guayaquil existen 115 gasolineras que pertenecen a 10 comercializadoras distintas. Las 3 comercializadoras más grandes son: PRIMAX COMERCIAL DEL ECUADOR S.A. Que responde a la marca "Primax", EXXONMOBIL ECUADOR CIA. LTDA. Que responde a la marca "Mobil" y LUTEXSA INDUSTRIAL COMERCIAL COMPAÑÍA LIMITADA que responde a la marca "Terpel" con $33 \%, 16 \%$ y $15 \%$ de la comercialización de combustibles respectivamente (segura, comunicación personal, 2015).

\section{Descripción de las partes de las gasolineras}

Las partes principales de una gasolinera para la comercialización de combustibles son los tanques de almacenamiento de gasolinas, medidores de flujo y dispensadores. Los primeros constituyen depósitos bajo tierra que sirven para contener la gasolina descargada por los autos tanques y los medidores de flujo sirven para medir el total de combustible que se introduce en el vehículo, a través de una computadora instalada en el dispensador que muestra la cantidad medida en litros. Finalmente el dispensador, o también llamado bomba de combustible, o surtidor es la herramienta utilizada para poner el combustible en el vehículo, para aquello cuentan con una manguera. Las mangueras suelen contar con un sistema que detecta cuando el tanque del vehículo ha sido llenado y detiene el suministro de combustible (Petróleos de Venezuela, S.A. [PDVSA], 2012). 
Adicionalmente, las gasolineras cuentan con otras herramientas de seguridad como las señaladas en la figura 2 y entre las más importantes tenemos:

- Marquesina: Cubierta ubicada sobre los dispensadores para protegerlos de la lluvia y sol.

- Bombas sumergibles: Equipo electromecánico de impulsión sumergido en el líquido almacenado en el tanque.

- Tuberías de venteo: Sistema diseñado para prevenir la formación de vacío o presión interna como consecuencia de llenados, vaciados o cambios de temperatura.

- Pozos de monitoreo: Pozos diseñados para monitorear el estado de las aguas subterráneas.

- Canaletas perimetrales: Conductos superficiales que llevan el agua contaminada con hidrocarburos hacia un sistema de tratamiento.

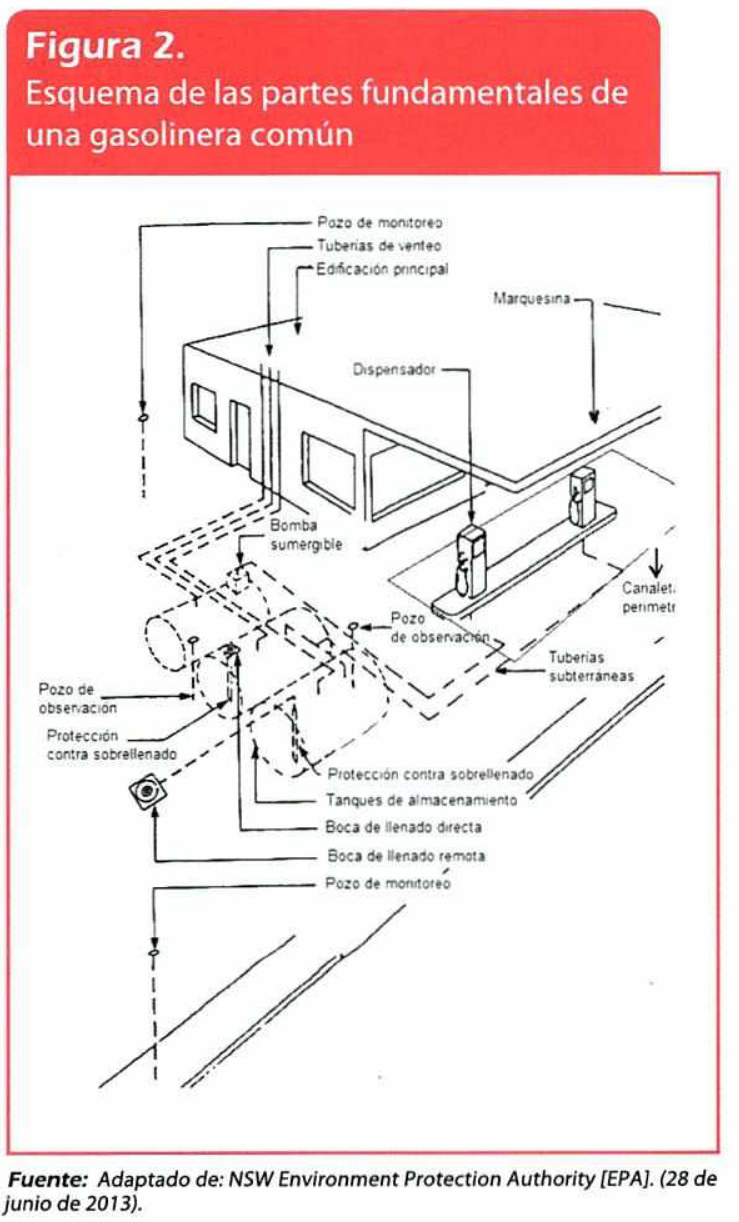

Descripción de la operación de las gasolineras

Recepción del producto

El encargado de la gasolinera controla la circulación interna de vehículos y otorga preferencia vial al auto tanque, mientras se estaciona en la zona de descarga, el mismo que como parte de las medidas de seguridad lleva inscrito en su parte posterior leyendas de seguridad como "PELIGRO INFLAMABLE", "CONSERVE SU DISTANCIA" (Ministerio de Energía y Minas, 1999).

La descarga de combustibles se realiza desde los autos tanques, hacia los tanques subterráneos de almacenamiento de la gasolinera. El combustible se descarga a través de mangueras flexibles con acoples herméticos, hasta las bocas de llenado de los tanques. Las bocas de llenado de combustible están pintadas de acuerdo al combustible que se almacena.

\section{Suministro de combustibles}

El suministro de combustibles a los clientes, se lo hace desde los surtidores electrónicos operados por los despachadores. Estos tienen un sistema automático para el control de llenado, que evita que se produzcan derrames de combustible. También disponen de una válvula de impacto que suspende el despacho cuando se ha producido un choque contra el surtidor.

Para el despacho de combustibles se siguen reglas de seguridad, como despachar únicamente a automotores que tengan su motor y aire acondicionado apagados y a vehículos de transporte público sin pasajeros. Es prohibido fumar en la estación o utilizar cualquier elemento que produzca chispa (Productos y Servicios Industriales C. LTDA., 2013).

\section{Normativa ambiental}

La normativa ambiental nacional que regula las operaciones hidrocarburíferas, incluyendo la operación de gasolineras, es el Reglamento Ambiental para las Opera- 
ciones Hidrocarburíferas en el Ecuador (RAOHE), expedido mediante el Decreto Ejecutivo No. 1215, Registro Oficial No 265 del 13 de febrero del 2001. Este reglamento cuenta con disposiciones generales para todas las operaciones hidrocarburíferas y específicas para cada actividad. La operación de las gasolineras estarían reguladas de manera específica por el capítulo $\mathrm{X}$ : Comercialización y venta de derivados de petróleo producidos en el país e importados. En este capítulo se indican disposiciones de seguridad tales como:

Los tanques para almacenamiento de líquidos combustibles e inflamables deben ser tanques horizontales, cilíndricos, atmosféricos, para instalación subterránea, con doble pared, provistos de un sistema de monitoreo intersticial de fugas, fabricados bajo estándares UL 58 y UL 1746.

Las líneas de venteo serán de 2 pulgadas de diámetro, cuya boca de descarga deberá estar a una altura no menos de 4 metros sobre el nivel de piso y estará provisto de una campana de venteo, para evitar el ingreso de aguas lluvias al tanque de almacenamiento.

En los surtidores que funcionan con bomba sumergible, deberá instalarse una válvula de emergencia, la cual deberá cerrarse automáticamente en el caso de que el surtidor sufra un golpe o volcamiento.

En las gasolineras no será permitido fumar ni hacer fuego, ni arrojar desperdicios y deberá contarse con la señalización correspondiente;

Junto a las bocas de descarga se instalará una toma a tierra, a la cual será conectado el auto tanque previo al trasvase del combustible, para eliminar la transmisión de la energía estática;

Por otra parte, para los programas de remediación se establecen criterios de remediación específicos. Los límites permisibles a aplicarse en un proyecto determinado dependerán del uso posterior a darse al suelo remediado. En la Tabla 1 se detallan los mismos.

\section{Tabla \# 1 \\ Limites permisibles para la identificación y remediación de suelos contaminados en todas las fases de la industria hidrocarburifera, incluidas las gasolineras}

\begin{tabular}{|c|c|c|c|c|c|}
\hline Parámetro & Expresado en & Unidad & $\begin{array}{c}\text { Uso } \\
\text { agrícola }\end{array}$ & $\begin{array}{l}\text { Uso } \\
\text { industrial }\end{array}$ & $\begin{array}{l}\text { Ecosistemas } \\
\text { sensibles }\end{array}$ \\
\hline Hidrocarburos totales & $\mathrm{TPH}$ & $\mathrm{mg} / \mathrm{kg}$ & $<2500$ & $<4000$ & $<1000$ \\
\hline $\begin{array}{l}\text { Hidrocarburos aromático } \\
\text { policíclicos (HAPs) }\end{array}$ & $\mathrm{s} \mathrm{C}$ & $\mathrm{mg} / \mathrm{kg}$ & $<2$ & $<5$ & $<1$ \\
\hline Cadmio & $\mathrm{Cd}$ & $\mathrm{mg} / \mathrm{kg}$ & $<2$ & $<10$ & $<1$ \\
\hline Níquel & $\mathrm{Ni}$ & $\mathrm{mg} / \mathrm{kg}$ & $<50$ & $<100$ & $<40$ \\
\hline Plomo & $\mathrm{Pb}$ & $\mathrm{mg} / \mathrm{kg}$ & $<100$ & $<500$ & $<80$ \\
\hline
\end{tabular}

Fuente: Decreto Ejecutivo 1215. (2 de febrero del 2001). Reglamento Sustitutivo del Reglamento Ambiental para las Operaciones Hidrocarburiferas en el Ecuador. Quito, Ecuador. 
El monitoreo consiste de una caracterización inicial del sitio y/o material a remediarse, un monitoreo de por lo menos un muestreo con los respectivos análisis cada seis meses y una caracterización final una vez concluidos los trabajos. Dependiendo de la tecnología de remediación aplicada, la frecuencia del monitoreo puede ser mayor.

\section{Derrames de hidrocatbutos}

A pesar de las medidas de seguridad establecidas para la operación de las gasolineras, la posibilidad de que se produzca un derrame al suelo sigue estando presente. De acuerdo al informe del Instituto Americano de Petróleo (2009), la fase de almacenamiento y consumo de combustibles es la segunda fase que más genera derrames con un $39,58 \%$, siendo superado por la fase de transporte con un $49,16 \%$.

Cuando se sospecha sobre la posibilidad de un derrame de hidrocarburos, se deben realizar evaluaciones ambientales en el sitio para diagnosticar y verificar el potencial de contaminación. La evaluación de impacto ambiental es una herramienta técnica que analiza los posibles efectos de las actividades humanas sobre el medio ambiente y presenta posibles soluciones que minimicen el impacto, para lo cual se la efectúa en tres fases.

La fase I de la evaluación ambiental del sitio representa una averiguación inicial que se limita a la investigación de registros históricos, escritos o electrónicos. De esta manera, se reúnen datos previos, actuales y en caso de que aplique futuros usos de la propiedad, su área de influencia y condiciones ambientales. Esta fase es de gran importancia, ya que es el punto de partida para las demás investigaciones y el diseño del futuro plan de remediación (Agencia de Protección Ambiental de los Estados Unidos [EPA], 1996).

Una vez que se han identificado las condiciones ambientales del sitio, se procede a la segunda fase conocida como evaluación ambiental donde se realiza una investigación más profunda. El objetivo de esta fase es confirmar la existencia de la contaminación, determinar su naturaleza y alcance. Para aquello, puede ser necesario realizar análisis de suelos, gases, aguas subterráneas y superficiales y sondear las posibles vías de migración de los contaminantes (EPA, 1996).

La fase III de la evaluación ambiental de sitio, incluye la remoción y disposición final del material contaminado, cumpliendo con la normativa ambiental (EPA, 1996).

\section{Descripción de derrames de derivados de petróleo}

Tanto el petróleo, como sus derivados están formados por una variedad de compuestos químicos. Estos compuestos incluyen hidrocarburos alifáticos, mismos que son prácticamente insolubles en agua; hidrocarburos aromáticos, que son muy solubles en agua; naftenos, que son poco solubles en agua, e hidrocarburos aromáticos poli-cíclicos que tienen una pobre solubilidad en agua. Además de los compuestos orgánicos mencionados, los derivados de petróleo también contienen oxígeno, azufre, nitrógeno, metales y otros elementos. $\mathrm{La}$ típica composición del petróleo está conformada por 57 a 63 vol. \% de hidrocarburos alifáticos, 3.5 a 7.5 vol. \% de naftenos y de 30 a 38 vol. \% de hidrocarburos aromáticos (Borowiec, Hoffman, Huculak, Rogowski \& Hoffman, 2008).

Entre los hidrocarburos aromáticos presentes en los derivados de petróleo están: benceno, tolueno y xileno, clasificados como los componentes más tóxicos del petróleo; debido a su alta solubilidad en agua, se disuelven en la misma y penetran con facilidad hacia aguas subterráneas. Los hidrocarburos aromáticos poli cíclicos son considerados carcinogénicos; penetran en el suelo, agua y atmosfera y se acumulan en el tejido adiposo de seres vivos. 
Los hidrocarburos alifáticos y naftenos se evaporan hacia la atmosfera, donde generan riesgo de explosión y de incendio si superan las concentraciones límites. Los naftenos en su mayor parte contaminan tanto suelos como aguas subterráneas, se evapora en el aire contenido en el suelo (Borowiec et al., 2008).

Cuando los combustibles se derraman en el suelo, una migración del contaminante vertical ocurre debido a las fuerzas capilares y gravitacionales. La fuerza de capilaridad también es responsable por la migración lateral (Konečný, Boháček, Müller, Kovářová \& Sedláčková, 2003).

La migración de los contaminantes en el suelo depende de una variedad de factores, entre los cuales tenemos: la concentración de los productos de petróleo, clima y varios parámetros del suelo como: tipo, capacidad de absorción, $\mathrm{pH}$, contenido de fase líquida, gaseosa y sólida. En el caso de suelos húmedos, cuando dominan temperaturas altas, los contaminantes de petróleo son biodegradados rápidamente, se evaporan, se mineralizan y se humidifican. En temperaturas bajas, los procesos de biodegradación son más lentos y la reducción de la concentración de contaminantes se daría principalmente por el proceso de difusión y de remojo en el suelo. Suelos arenosos tienen una alta permeabilidad, lo cual favorece la separación de fases de los productos de petróleo y la migración de gases hacia la superficie (Borowiec et al., 2008).

\section{Descripción del sitio}

El cantón Guayaquil ocupa 482.016,83 ha de superficie intervenida (MAGAP, 2011). Siendo uno de los cantones más importantes en Ecuador, debido a que sus actividades productivas representan más del $20 \%$ de la economía de todo el país; por lo que es una de las más grandes áreas urbanas y está sumamente industrializada, ubicada en la zona estuarina de la parte baja y margen derecho del Río Guayas. De manera general, la estratigrafía del suelo consiste de profundos sedimentos blandos sobre rocas duras del periodo Cretácico. Estos sedimentos delta estuarinos se depositaron durante un ambiente salobre y son inusualmente débiles y altamente compresibles. El grosor de este sedimento varía en distintas partes de la ciudad ente 15 y 60 metros; debido a su proximidad con el río tiene un nivel freático muy alto (VeraGrunauer, Bray, Pestana, Kayen, Tandazo, Ramírez, Vera-Grunauer, \& Mera-Ortiz, 2006).

Por sector se tiene que el centro de la ciudad tiene un suelo blando y de arcilla blanca. En el norte y sur de la ciudad el suelo es más duro y se asienta en su mayor parte sobre rocas sedimentarias y calizas (El Universo, 2010).

Según el sistema americano de Clasificación de Suelos denominado "Soil Taxonomy, USDA, 1975", los suelos predominantes en la zona costera húmeda sur, que incluye al cantón Guayaquil, son inceptisoles (Ministerio de Agricultura, Ganadería, Acuacultura y Pesca, 2002) y molisoles (Ibáñez, 2008). Tanto los inceptisoles como molisoles son suelos derivados de materiales de naturaleza volcánica y sedimentaria. Son superficiales, moderadamente profundos y de topografía plana a quebrada (Department of Sustainable Development (DSD), s.f.)

Además, son llamados suelos inmaduros porque son jóvenes y con escasa expresión morfológica. La textura de estos suelos puede ser predominantemente limo-arcillosos en la superficie y totalmente arcillosos en profundidad; además de contar con un buen drenaje (Torres \& Zuluaga, 2009).

\section{Perspectivas para el tratamiento}

Los suelos contaminados pueden ser remediados mediante distintos métodos. La remediación de un sitio usualmente incluye 
el tratamiento, tanto del suelo como el del agua subterránea contaminada (Gente \& Lausdei, 2012). Existen tres estrategias principales que pueden ser utilizadas individualmente, o en conjunto para remediar la mayoría de sitios contaminados:

La primera es la destrucción, o alteración de contaminantes mediante la alteración de su estructura química y pueden dividirse en métodos térmicos, biológicos, o químicos que a su vez pueden ser aplicados in situ o ex situ. Segundo, la extracción o separación de contaminantes que incluye: desorción térmica, lavado de suelos, extracción con disolvente y la extracción del vapor del suelo y tratamiento de las aguas subterráneas por separación de fases, adsorción con carbón, separación por aire, intercambio iónico, o cualquier combinación de las mencionadas anteriormente. Finalmente, la inmovilización de contaminantes que incluye: la estabilización, solidificación y tecnologías de contención como colocar rellenos de seguridad o muros.

Para la estrategia de alteración y separación de contaminantes, existen tres tipos de técnicas: térmicas, fisicoquímicas y biológicas. Entre las térmicas se destacan, la desorción térmica y la incineración.

La desorción térmica consiste en calentar el suelo contaminado con contaminantes orgánicos, con el fin de vaporizarlos y separarlos del suelo. Posteriormente los contaminantes deben ser dirigidos hacia un sistema de tratamiento de gases. Se aplica para separación de compuestos orgánicos de desechos, así como para suelos contaminados con creosota e hidrocarburos. Se la considera una técnica de corto a largo plazo y tiene un costo aproximado de $350 \mathrm{USD} /$ ton. $\mathrm{La}$ presencia de cloro en el contaminante es una limitación para el desarrollo de esta técnica, ya que puede afectar la volatilización de algunos metales, como el plomo. No es efectiva en zonas saturadas, suelos muy compactos o con permeabilidad variable.
Además, producen emisiones gaseosas.

En la incineración se utilizan altas temperaturas de operación que van desde los 870 a los $1200^{\circ} \mathrm{C}$ para volatilizar y quemar compuestos orgánicos en presencia de oxígeno. La efectividad de remoción y destrucción adecuada excede el $99 \%$. Sin embargo, los gases de combustión generalmente requieren de tratamiento y se la considera una técnica de corto a largo plazo con un costo entre 200 y 1000 USD/ton. Efectos negativos son los metales pesados que pueden producir cenizas que requieren estabilización, o podrían reaccionar con otros compuestos formando compuestos más volátiles y tóxicos.

Entre las fisicoquímicas se destacan la fase de extracción de fase dual y oxidación química in situ. La extracción de fase dual emplea un sistema de alto vacío para eliminar varias combinaciones de las aguas subterráneas contaminadas, productos derivados del petróleo en fase separada y vapores de hidrocarburos del subsuelo. Los vapores y líquidos extraídos son tratados y eliminados por medio del sistema de alto vacío. Es usada a menudo para remediar sitios contaminados por derrames o fugas de $\operatorname{COV} y$ algunas gasolinas. Además facilita la inducción de procesos de bio remediación. Puede tardar desde días a años, dependiendo de la cantidad de combustible y puede costar $80 \mathrm{USD} /$ ton. el aire y líquido deben ser tratados o dispuestos.

La oxidación química in situ consiste en la oxidación de los compuestos de hidrocarburos clorados por otro agente químico activo. Ataca principalmente a compuestos inorgánicos. Puede ser utilizado con menos eficiencia para COV no halogenados, gasolinas y pesticidas. Se la considera una técnica de corto a mediano plazo y tiene un costo entre 190 y 600 $\mathrm{USD} / \mathrm{m} 3$. Puede ocurrir una oxidación incompleta o formación de compuestos intermediarios. La presencia de aceites y 
grasas disminuye la eficiencia.

Entre las biológicas se destacan la bioaumentación y la atenuación natural. La primera consiste en la adición de microorganismos vivos para degradar, o transformar compuestos orgánicos tóxicos a otros menos tóxicos o inocuos. Ha sido utilizada con éxito para tratar suelos, lodos y sedimentos contaminados con hidrocarburos del petróleo. Puede tardar varios meses o años y tiene un costo aproximado de $100 \mathrm{USD} /$ ton. Antes de llevar a cabo el proceso de remediación, deben realizarse cultivos de enriquecimiento, aislar micro-organismos capaces de metabolizar el contaminante y cultivarlos hasta obtener grandes cantidades de biomasa.

La atenuación natural consiste en procesos físicos, químicos y biológicos no aumentados que actúan para limitar la migración y reducir la concentración de contaminantes en el subsuelo. Es utilizada cuando es técnicamente inviable limpiar un sitio afectado por hidrocarburos hasta los límites máximos permisibles establecidos en lo normal. Es una técnica a largo plazo y de bajo costo que se emplea para todo tipo de suelo con hidrocarburos, incluyendo los existentes en las ciudades. Una de sus desventajas es el riesgo potencial que corren los receptores durante el tiempo que dura la atenuación.

Cuando el uso de una tecnología no logra la restauración del sitio deseada, es posible que se deba recurrir a un tren de tratamiento. Este se refiere al uso de dos o más tecnologías juntas que actúan como procesos integrados, o se combinan en secuencia (Volke \& Velasco, 2002).

\section{Metodología}

El área seleccionada para el estudio se delimitó dentro del perímetro urbano del cantón Guayaquil, donde se realizó observaciones en 43 gasolineras y se evidenció que las mismas están ubicadas en las avenidas principales de la ciudad; así se observa la mayor parte del tiempo; y consta en la Figura 3, mismas que fueron colectados entre junio y julio del 2015.

\section{Figura 3.}

Mapa de ubicación de las gasolineras muestreadas en el cantón Guayaquil.
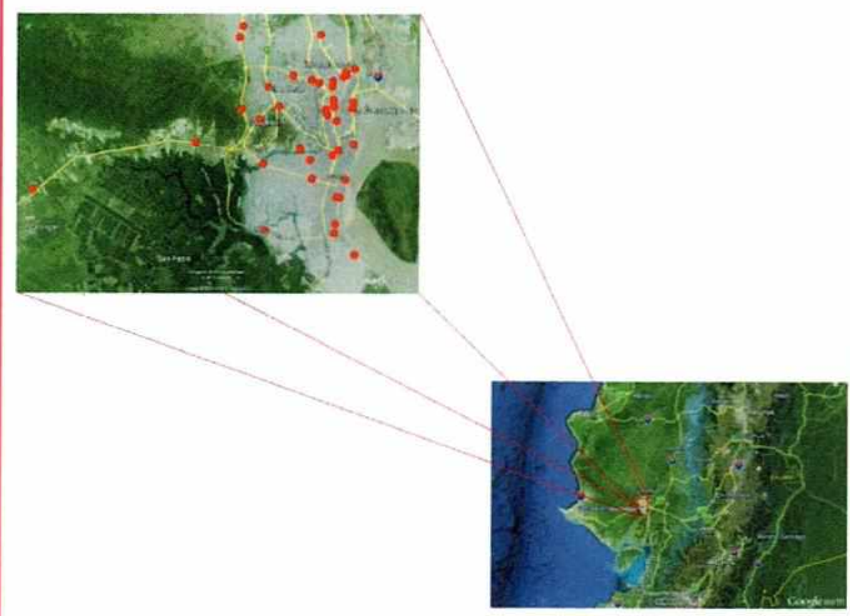

La investigación fue de tipo exploratoria - descriptiva debido a que el problema de estudio no ha sido ampliamente abordado en investigaciones anteriores, ya que la comercialización de combustibles es un tema sensible en Ecuador, debido al manejo deficiente de las gasolineras (Cortázar, 2013).

La población fue el total de gasolineras dentro del perímetro urbano $\mathrm{N}=115$ y el tamaño de la muestra utilizada fue de 43 gasolineras correspondiente al $37 \%$ del total. La gasolinera resultó ser la unidad de muestreo donde se registraron las siguientes variables: 
- Combustibles comercializados

- Número de marquesinas

- Número de islas de despacho

- Número de tanques de almacenamiento

- Capacidad total de almacenamiento

- Presencia de canaletas perimetrales en la marquesina y en el área de tanques

- Presencia de señalética de seguridad en área de tanques

- Presencia de pozos de monitoreo de aguas subterráneas.

Para la obtención de datos del enfoque cualitativo se utilizó la técnica de entrevistas a expertos. Se eligieron 4 individuos expertos de cada sector social del objeto de estudio: un servidor público de la Agencia de Regulación y Control Hidrocarburífero $(\mathrm{ARCH})$, un consultor ambiental de proyectos de remediación, un gerente de una gasolinera y un jefe de control y seguimiento ambiental del Gobierno Provincial del Guayas (GPG). Las preguntas de las entrevistas fueron específicas para cada experto, de acuerdo a la función que desarrollan.

\section{Análisis de los datos}

Para el análisis de los datos cuantitativos se utilizaron tablas de frecuencia, medidas de tendencia central (e.g. moda y mediana), medidas de dispersión (e.g, desviación estándar), e histogramas para la presen- tación de datos y distribuciones de frecuencias. El análisis de los datos cualitativos consistió en la agrupación de respuestas comunes.

\section{Resultados y discusión}

Los resultados de las inspecciones realizadas a las tres comercializadoras de combustibles más grandes de Guayaquil: Primax, Mobil y Terpel indican que los tipos de combustibles de mayor presencia en las gasolineras son ecopaís, super y diésel, como se puede observar en la figura 4A. Esto se debe a que el parque automotor de la zona urbana consume fundamentalmente estos combustibles (Kogan, 2015). El combustible menos ofertado es el GLP debido a que solo debe ser despachado a automóviles que cuenten con el sistema apropiado para su funcionamiento y la oferta de estos sistemas es muy limitada actualmente y es únicamente para taxis (Agencia Pública de Noticias del Ecuador y Suramérica ANDES, 2014).

Las marquesinas que son utilizadas para proteger los surtidores de la influencia de la lluvia y sol normalmente se encontraron a razón de una por gasolinera. La moda de la cantidad de surtidores por gasolinera es cuatro y en su generalidad uno corresponde al expendio de diésel y el resto son surtidores que combinan el despacho de super y ecopaís, ver figura 4B.

\section{Figura 4. \\ Tipos de combustibles expedidos en gasolineras y número de surtidores por gasolineras en \\ Guayaquil, (A) Combustibles comercializados, (B) Número de dispensadores de combustible}

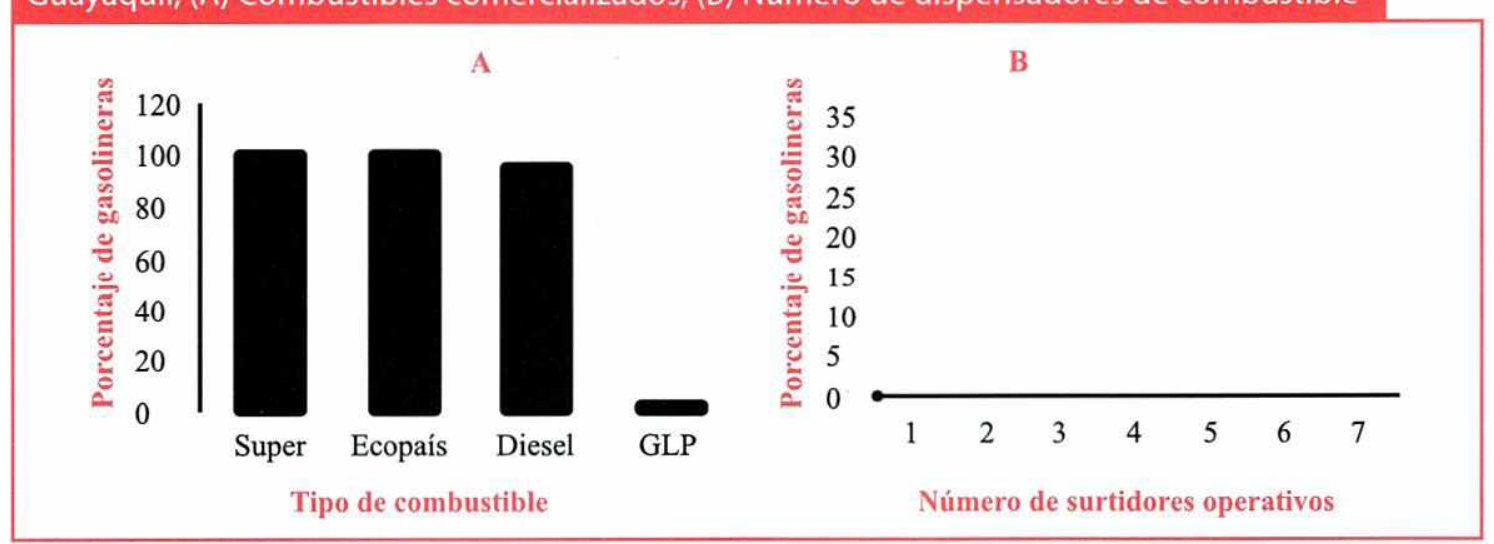


Los números de tanques más comunes son tres y cuatro, con una capacidad que va desde 7.000 a 12.000 galones por tanque, ver figura $5 \mathrm{~A}$. La capacidad total de almacenamiento de combustible por gasolinera está entre 10.000 y 60.000 galones. La moda de la capacidad total de almacenamiento cae en el rango de 40.000 a 50.000 , ver figura 5B. La capacidad de almacenamiento promedio es 36.514 galones, con una desviación estándar de 12.352 galones.

El $95 \%$ y $100 \%$ de las gasolineras cuentan con canaletas perimetrales superficiales en el área de surtidores y en el área de tanques respectivamente. Por lo tanto se concluye que se cuentan con las herramientas para conducir aguas contaminadas con hidrocar- buros hacia un sistema de tratamiento.

En el área de tanques, el $88 \%$ de las gasolineras cuentan con señalética de seguridad que indica las recomendaciones al momento de descargar combustibles en los tanques subterráneos. La señalética de seguridad propone medidas para prevenir accidentes que afectarían la salud de personas y la calidad del ambiente.

El $100 \%$ de las gasolineras cuentan con pozos de monitoreo de aguas subterráneas. Los pozos de monitoreo son una importante herramienta, que permite a los trabajadores de las gasolineras verificar la presencia de derrames de combustibles en el subsuelo.

\section{Figura 5.}

(A) Número de tanques subterráneos, (B) Capacidad total de almacenamiento en galones por gasolinera.

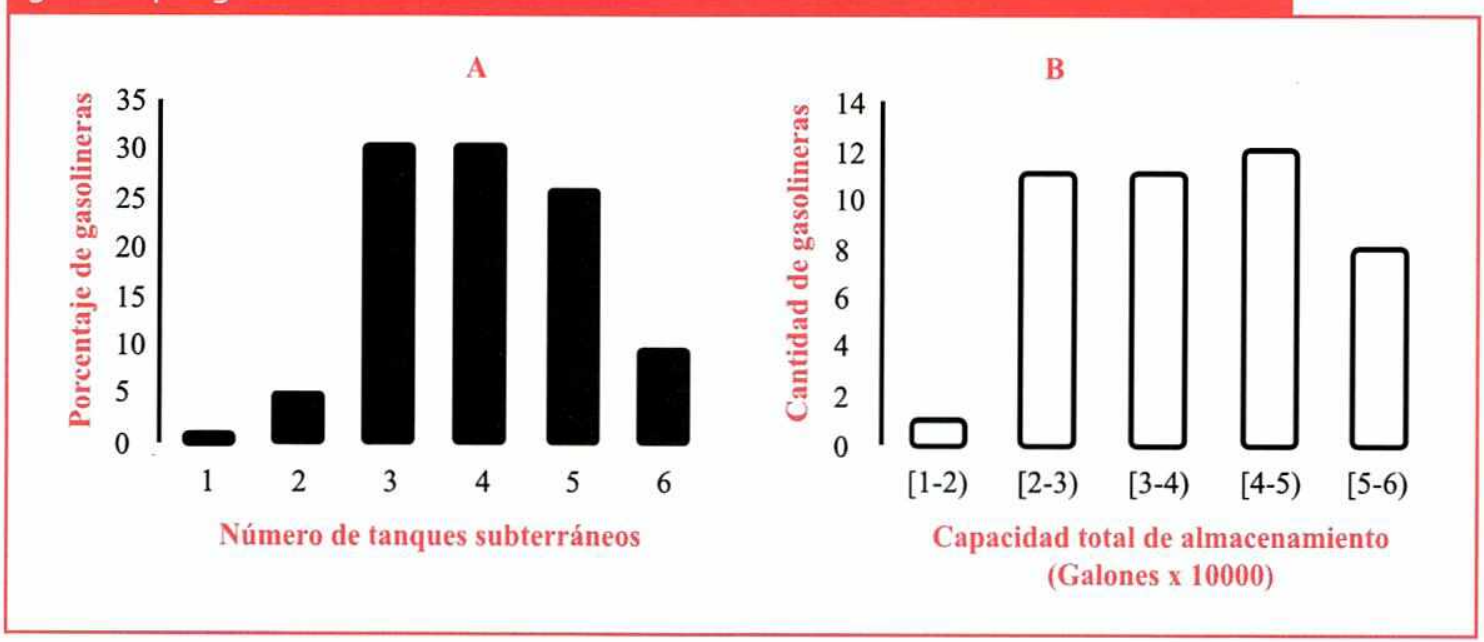

Como resultado de las entrevistas a expertos, se obtuvo que considerando que los tanques de almacenamiento de combustibles se encuentren enterrados, no se puede detectar un derrame a simple vista.

Cuando ha ocurrido un derrame una de las posibles evidencias es problema de olores en los sectores cercanos a la gasolinera (Segura, comunicación personal, 2015). Además, todas las gasolineras deben tener obligatoriamente pozos de monitoreo que sirven para verificar la presencia de combustibles en aguas subterráneas (Torres, comunicación personal, 2015).

Adicionalmente, existen sistemas de medición automática y detección de fugas como la consola Veeder Root. De esta manera, se monitorean constantemente los niveles de combustibles en los tanques y se los compara con las ventas. El sistema 
arroja una alarma en caso de pérdidas repentinas (Mendoza, comunicación personal, 2015). Otro método para determinar si se pudo haber incurrido en un derrame, es la revisión de los estudios de mantenimiento de los tanques. En los mismos, se puede evidenciar si es que hay fisuras, o perforaciones en los tanques que conllevarían a un derrame (Erazo, comunicación personal, 2015).

No existe un volumen específico que determine si un derrame es significativo, o meritorio de intervención e incluso cantidades mínimas, como un litro de combustible en el suelo, deben ser contenidos. Sin embargo, cuando ocurren derrames en volúmenes suficientemente grandes como para afectar grandes zonas, e incluso aguas subterráneas, se debe realizar una evaluación ambiental que determine la magnitud y forma de la mancha (Erazo, comunicación personal, 2015).

Entre los principales factores que pueden ocasionar un derrame en el suelo, están los años de servicio de los tanques y la calidad de los materiales de los mismos (Mendoza, comunicación personal, 2015). Se conoce que muchas de las gasolineras en Ecuador existen desde antes de 1995 (La Hora, 2012). En Estados Unidos, un estimado de un millón de tanques subterráneos tienen más de 16 años y se estima que alrededor del $25 \%$ del total de tanques estarían filtrando (Ministerio de Energía y Minas, s.f.).

Al ocasionarse un derrame y al momento de decidirse por una tecnología de remediación, una de las prioridades para los gerentes de las gasolineras es que no se destruya la infraestructura en la que ellos han invertido y que todo plan se desarrolle con el menor impacto posible sobre su construcción. Utilizando una tecnología adecuada, es posible lograr una restauración completa del sitio y definitivamente llegar a los niveles máximos permisibles que disponen las autoridades competentes (Torres, comunicación personal, 2015).

Las metodologías más utilizadas en gasolineras de la ciudad son los físicos químicos como reposición del suelo, extracción del suelo y descontaminación, o se entrega el suelo contaminado a un gestor para su tratamiento o disposición final. Estos métodos pueden ser utilizados en conjunto con la inoculación de bacterias que degradan el combustible restante (Erazo, comunicación personal, 2015).

Es importante recalcar que métodos físicos químicos podrían verse limitados en caso de que existan tuberías pasando por el área contaminada. Es posible que en estos casos, se deba recurrir a un tren de tratamiento. En otras investigaciones como la realizada por Suresh, Jawitz, Enfield, Falta, Annable \& Lynn (2002), se indica que para tratar sitios contaminados existe una necesidad de integrar tecnologías para llegar a un tratamiento con una alta razón efectividad/costo.

Los expertos entrevistados coincidieron en que los criterios más importantes para elegir una técnica de remediación son el tiempo y la eficacia; así como las características propias del sitio. La opinión de los expertos es concordante con lo indicado por Volke \& Velasco en el libro Tecnologías de remediación para suelos contaminados (2002).

No obstante, mayores investigaciones que demuestren la efectividad del uso combinado de ciertas tecnologías de remediación, serían beneficiosas en el ámbito de contaminación del suelo por derrames de hidrocarburos en la ciudad. Además como continuación de este trabajo, sería conveniente verificar hasta qué punto las herramientas para el control de derrames en las gasolineras de Guayaquil son efectivas para prevenir o detectar los mismos. 


\section{Conclusión y recomendaciones}

Considerando el número de surtidores en las gasolineras de la ciudad de Guayaquil que se ubican en las avenidas principales y que cuentan en su mayoría con una sola marquesina que protege entre 4 y 6 surtidores de combustible, se estimaría que son de tamaño mediano; aunque, esta categoría dada es propia de la investigación.

En promedio se almacenan aproximadamente 36.514 galones de combustible por gasolinera y están distribuidos en su mayor parte de 3 a 5 tanques de almacenamiento; un galón de petróleo o sus derivados puede contaminar un millón de galones de agua y una pequeña fisura en cada tanque puede derramar más de 400 galones de combustible al año (Sierra Club, s.f.). Con esta consideración, podemos relacionar la magnitud de un posible derrame en los tanques de las gasolineras en Guayaquil.

Se encontró que las gasolineras en Guayaquil cuentan con herramientas para prevenir y detectar derrames de combustibles en la superficie, tales como canaletas perimetrales y en el subsuelo, la señalética de seguridad, mantenimiento de los tanques de almacenamiento, pozos de monitoreo y sistemas de detección de fugas.

Los actores sociales relacionados con la actividad de comercialización de combustibles, que incluyen a las gasolineras y sus representantes, las autoridades competentes y consultores ambientales, cuentan con herramientas y procedimientos establecidos para la gestión y control de derrames de hidrocarburos.

A pesar de tener estas herramientas, los derrames de combustibles en el subsuelo ocurren y necesitan ser remediados. Una de las preocupaciones de los gerentes de las gasolineras es el daño en la infraestructura, que la ejecución de un plan de remediación. En general, se encontró que los criterios más importantes al momento de elegir una técnica de remediación son: eficacia y tiempo. Otras variables como: estructura geológica del suelo, planos as built, puntos de afectación peligrosos alrededor de la gasolinera, cantidad y tiempo del derrame también son importantes pero muy específicas de cada sitio.

Considerando el alto nivel freático del cantón Guayaquil, se prevé que la remediación debe ser, tanto del suelo como del agua subterránea. Los métodos más utilizados en estos casos son los físico, químicos, que incluyen la extracción física del suelo y agua contaminada y su posterior tratamiento.

\section{Referencias bibliográficas}

Agencia de Protección Ambiental de los Estados Unidos. (1996). Evaluación de Sitios. En Rutas a Tecnologías para la Investigación y Limpieza de Terrenos Contaminados (págs. 21-34). Washington DC.

Agencia de Protección Ambiental de los Estados Unidos. (1996). Investigación de sitios. En Rutas a tecnologías para la investigación y limpieza de terrenos contaminados (págs. 35-52). Washington DC.

Agencia de Regulación y Control Hidrocarburífero. (05 de noviembre del 2015). Reglamento Actividades de Comercialización de Derivados del Petróleo, expedido mediante Registro Oficial Suplemento 621. Quito.

Agencia Pública de Noticias del Ecuador y Suramérica ANDES. (1 de enero del 2014). Taxistas del sur ecuatoriano proponen cambiar su combustible por gas natural. Recuperado el 25 de agosto de 2015: http://www.andes.info.ec/ fr/node/27063 
American Petroleum Institute. (2009). Analysis of U.S. Oil Spillage. New York: Environmental Research Consulting. API Publishing Services. Washington. USA. Págs. 86.

Borowiec, M., Hoffman, K., Huculak, M., Rogowski, S., \& Hoffman, J. (2008). Effect of petrol stations on the contamination of the soil environment. Wydawnictwo Politechniki Krakowskiej, 62-68.

Cerón Hidalgo, H. G. (2010). Estudio para la implementación de una estación de servicio en la ciudad de Sangolquí: Escuela Superior Politécnica del Ejército (ESPE).

Cortázar, J. L. (26 de agosto del 2013). De la extinción de gasolineras al control de los subsidios. (Diario El Telégrafo, entrevistador)

Decreto Ejecutivo 1215. (02 de febrero del 2001). Reglamento Sustitutivo del Reglamento Ambiental para las Operaciones Hidrocarburiferas en el Ecuador. Quito, Ecuador.

Department of Sustainable Development (DSD). (s.f.). Organization of American States. Obtenido de Clasificación taxonómica de los suelos identificados: http://www.oas.org/dsd/publications/ unit/oea30s/ch026.htm

Dirección de análisis estadístico $\mathrm{y} / \mathrm{o}$ indicador. (2015). Compendio de indicadores de resultado de los sectores estratégicos. Ministerio Coordinador de Sectores Estratégicos.

El Universo. (22 de agosto del 2010). Suelo de Guayaquil incrementa su vulnerabilidad ante un sismo. Recuperado el 25 de agosto del 2015, de El Universo: http://www.eluniverso.com/2010/08/22/ 1/1445/suelo-guayaquilincrementa-vulnerabilidad-ante-un-sismo.html
Erazo, J. C. (julio de 2015). Manejo de estaciones de servicio. (M. V. Vaca, Entrevistador)

Gente, V., \& Lausdei, D. (2012). A Matrix Based Approach for Modelling of Treatment Processes for Contaminated Groundwater. Separating Pro-Environment, 123-138.

Ibáñez, J. J. (2 de marzo del 2008). Fundación para el Conocimiento Madrimasd. Recuperado el 25 de agosto del 2015, de Suelos de Perú y Ecuador: http://www. madrimasd.org/blogs/universo/2008/03/ $02 / 85725$

Kogan, E. (13 de julio del 2015). Vale la pena considerar un auto diésel. $L a$ Opinión.

Konečný, F., Boháček, Z., Müller, P., Kovářová, M., \& Sedláčková, I. (2003). Contamination of soils and groundwater by petroleum hydrocarbons and volatile organic compounds - Case study: ELSLAV BRN. Bulletin of Geosciences, 225-239.

La Hora. (16 de octubre del 2012). Gasolineras: Riesgo está bajo tierra. La Hora.

López, F., Santana, K., Rodríguez, M., \& Mesa, D. (s.f.). Estudio de caso: remediación de suelos contaminados por hidrocarburos volátiles en una estación de servicio en Bogotá D.C. Entérese, 17-23.

Lucas, R., Vázquez, S., Lo Balbo, A., \& Mac Cormack, W. (s.f.). Biorremediación de suelos contaminados con hidrocarburos utilizando bacterias antárticas sicrotolerantes.

Mendoza, A. (agosto del 2015). Medidas de seguridad de gasolineras. (M. V. Vaca, Entrevistador) 
Ministerio de Agricultura, Ganadería, Acuacultura y Pesca (MAGAP). (2011). Memoria Técnica: Evaluación de tierras por su capacidad de uso. Guayaquil.

Ministerio de Agricultura, Ganadería, Acuacultura y Pesca (MAGAP). (2002). Mapa de Suelos del Ecuador - Taxonomía.

Ministerio de Energía y Minas. (1999). Reglamento de Operación y Seguridad del Transporte Terrestre de Combustibles (Excepto el GLP) en auto tanques. Quito.

Ministerio de Energía y Minas. (s.f.). Guía Ambiental Para el Manejo de Tanques de Almacenamiento Enterrados. Lima.

Ministerio del Ambiente. (2011). Estudio para conocer los potenciales impactos ambientales y vulnerabilidad relacionada con las sustancias químicas y tratamiento de desechos peligrosos en el sector productivo del Ecuador.

NSW Environment Protection Authority (EPA). (28 de junio del 2013). Service station sites: assessment \& remediation. Obtenido de http://www.epa.nsw.gov. $\mathrm{au} / \mathrm{mao} /$ servicestation.htm

Petróleos de Venezuela, S.A. (PDVSA). (25 de noviembre del 2012). Aprendamos del Petróleo: Estación de Servicio. Ciudad Caracas.

Productos y Servicios Industriales C. LTDA. (mayo del 2013). Gobierno Provincial del Guayas. Recuperado en agosto del 2015, de Estudio de Impacto Ambiental Ex-Post Estación de Servicio Eloy Alfaro Durán: http://www.guayas.gob. ec/dmdocuments/medio-ambiente/eia/ 2013/2013-agosto/EIA\%20Ex-Post \%20 ELOY\%20ALFARO\%20DURAN.pdf

Revista Vistazo. (2013). 500 Mayores empresas del Ecuador. Vistazo.
Segura, D. (18 de agosto del 2015). Manejo de estaciones de servicio en Guayaquil. (M.V. Vaca, Entrevistador)

Sierra Club. (s.f.). Leaking Underground Storage Tanks: A Threat to Public Health \& Environment. San Francisco.

Suresh, P., Jawitz, J., Enfield, C., Falta, R., Annable, M., \& Lynn, A. (2002). Technology integration for contaminated site remediation: clean-up goals and performance criteria . Groundwater Quality: Natural and Enhanced Restoration of Groundwater Pollution, 571-578.

Torres Delgado, K., \& Zuluaga Montoya, T. (2009). Bioremediación de suelos contaminados por hidrocarburos. 2009: Universidad Nacional de Colombia.

Torres, F. (agosto de 2015). Planes de Remediación. (M. V. Vaca, Entrevistador)

Van Wezel, A. (2007). Social cost-benefit analysis of the soil remediation operation in the Netherlands. Holanda: The Netherlands Environmental Assessment Agency.

Vera-Grunauer, X., Bray, J., Pestana, J., Kayen, R., Tandazo, E., Ramírez, J., Vera-Grunauer J.G. \& Mera-Ortiz W. (2006). Site characterization and seismic zonation of Guayaquil City, Ecuador. Proceedings of the 8th U.S. National Conference on Earthquake Engineering. Paper No. 1467. California, USA.

Volke Sepulveda, T. \& Velasco, J. A. (2002). Tecnologías de remediación para suelos contaminados. México: Instituto Nacional de Ecología (INE-SEMARNAT).

Yépez, F. (18 de febrero del 2013). Economía Random. Recuperado el 15 de agosto del 
2015, de El subsidio más grande del Ecuador no es para los más pobres: https://economiarandom.wordpress.co $\mathrm{m} / 2013 / 02 / 18 /$ el-subsidio-mas-grande-d elecuador-no-es-para-los-mas-pobres/

Zamora, A., Ramos, J., \& Arias, M. (2012). Efecto de la contaminación por hidrocarburos sobre algunas propiedades químicas y biológicas de un suelo de sabana. Bioagro, 5-12.

\section{Maria Victoria Vaca Molina}

Ingeniera en Gestión Ambiental. Facultad de Artes Liberales, Escuela de Ciencias Ambientales. Universidad Espíritu Santo - Ecuador.

E-mail: marivivaca@uees.edu.ec. vaca.victoria92@gmail.com

\section{Evelyng Astudillo Sainchez}

Bióloga.

Magister en Espacios Naturales Protegidos. Docente tiempo completo de la Facultad de Artes Liberales, Escuela de Ciencias Ambientales de la Universidad Espiritu Santo - Ecuador.

E-mail: eastudillo@uees.edu.ec 